TRANSACTIONS OF THE

AMERICAN MATHEMATICAL SOCIETY

Volume 352, Number 5, Pages 2407-2427

S 0002-9947(00)02281-9

Article electronically published on February 16, 2000

\title{
REPRESENTATIONS OVER PID'S WITH THREE DISTINGUISHED SUBMODULES
}

\author{
STEVE FILES AND RÜDIGER GÖBEL
}

\begin{abstract}
Let $R$ be a principal ideal domain. The $R$-representations with one distinguished submodule are classified by a theorem of Gauß in the case of finite rank, and by the "Stacked Bases Theorem" of Cohen and Gluck in the case of infinite rank. Results of Hill and Megibben carry this classification even further. The $R$-representations with two distinguished pure submodules have recently been classified by Arnold and Dugas in the finite-rank case, and by the authors for countable rank. Although wild representation type prevails for $R$-representations with three distinguished pure submodules, an extensive category of such objects was recently classified by Arnold and Dugas. We carry their groundbreaking work further, simplifying the proofs of their main results and applying new machinery to study the structure of finite- and infinite-rank representations with two, three, and four distinguished submodules. We also apply these results to the classification of Butler groups, a class of torsion-free abelian groups that has been the focus of many investigations over the last sixteen years.
\end{abstract}

\section{INTRODUCTION}

The abundance of torsion-free abelian groups [18, 19, 20, 29] indicates that it is hopeless to classify them; any sort of classification of torsion-free groups must inevitably be carried out under rather severe restrictions.

Well-known examples are limitations to finitely generated groups or to groups of rank 1 , that is to say to subgroups of $\mathbb{Q}$, see 29]. Classification theorems follow the usual strategy. First we find a result showing that the groups of the class under consideration can be decomposed into direct sums of indecomposables. This is possible for many rings $R$ and $R$-modules of finite rank.

Assuming that the "building blocks"-indecomposable modules-determine the module uniquely (which is often the case), it then remains to classify the indecomposable modules, which is possible if there are not too many of them. A good candidate for such objects are Butler groups with small typesets. Fortunately these torsion-free groups of finite rank can be seen as images under a functor, called the Butler functor, taking representations $\mathbf{F}=\left(F, F_{0}, F_{1}, \ldots, F_{n-1}\right) \in \operatorname{Rep}_{n} R$ of free

Received by the editors November 20, 1996 and, in revised form, October 3, 1997.

2000 Mathematics Subject Classification. Primary 16G60, 13C05, 20K15, 20K20; Secondary 20K25, 20K40, 15A36.

Key words and phrases. Modules with distinguished submodules, decomposition into indecomposables, Butler groups, classification theorems.

Supported by the Graduierten Kolleg Theoretische und experimentelle Methoden der reinen Mathematik of Essen University and a project No. G-0294-081.06/93 of the German-Israeli Foundation for Scientific Research \& Development. 
$R$-modules $F$ over principal ideal domains $R$ with $n$ distinguished submodules $F_{i}$ to certain torsion-free abelian groups, see [4, 5, 8, 11, 13, 14, 15, 16]. The crucial point is that this Butler functor preserves direct sums. Hence the indicated decomposition problem of Butler groups can be transported to $R e p_{n} R$, which is studied independently.

Earlier results [5, 8, 12, 23, 33, 34, 48 and the discussion in 33, for instance, show that classification in $R e p_{n} R$ is possible only for $n \leq 4$. If $R$ is a field and $n \leq 4$ this is now well-known, see [48. However if $R$ is a PID and not a field new complications arise and classification may be hopeless even for $n=2$, witness [23. The key observation is the fact that classification strongly depends on the kinds of embeddings of the $F_{i}$ 's in $F$. It helps substantially if the $F_{i}$ 's are pure submodules of $F$ for $\mathbf{F}=\left(F, F_{0}, F_{1}\right) \in \operatorname{Rep}_{2} R$, a condition which trivially holds for fields. However, we can still find many $R_{2}$-modules $\mathbf{F}$ (elements in $R e p_{2} R$ ) of this sort, of rank $\aleph_{0}$, with $F_{0} \cap F_{1}=0$ and End $\mathbf{F}=\mathbb{Z}$; see [28] and [23]. Such an abundance of indecomposable $R_{2}$-modules of infinite rank shows that a classification theorem needs further restrictions. In 28 , when we impose that $F / F_{0} \oplus F_{1}$ is a bounded $R$-module, we are able to write $R_{2}$-modules of at most countable rank as direct sums of (a list of) indecomposable $R_{2}$-modules of rank $\leq 2$.

In order to deal with $R_{3}$-modules, we observe that their structure is even more complex, and classification needs further restrictions.

We say that $\mathbf{F}=\left(F, F_{0}, F_{1}, \ldots, F_{n-1}\right) \in R e p_{n} R$ is a complemented representation if $F$ is a free $R$-module, all the $F_{i}$ 's are pure in $F, F_{0}+F_{1}=F$, and $F_{i} \cap F_{j}=0$ for all $i \neq j$. Of course, we say that an object $\mathbf{F} \in R e p_{n} R$ has finite rank if the free $R$-module $F$ has finite rank.

Later in the paper (Example 4.8), we will note the existence of a complemented representation $\mathbf{F}=\left(F, F_{0}, F_{1}, F_{2}\right)$ of countably infinite rank which is not a direct sum of finite rank $R_{3}$-modules, but which is such that all factors $F / F_{i} \oplus F_{j}(i \neq j)$ are nevertheless direct sums of cyclic $p$-modules, one for each prime $p$ in $R$. In order to avoid the obvious difficulties encountered in this example, we will require that $\mathbf{F}$ has finite rank. Now we are successful in obtaining the desired decomposition result (Theorem 2.6):

Let $\mathbf{F}=\left(F, F_{0}, F_{1}, F_{2}\right) \in$ Rep $_{3} R$ be a complemented representation of finite rank. Then $\mathbf{F}$ is a direct sum of complemented $R_{3}$-modules of rank $\leq 2$.

The indecomposable complemented $R_{3}$-modules are listed as Remark 2.7

From this, our main classification result concerning $\operatorname{Rep}_{3} R$, we derive a number of results about representations with two, three and four submodules, as well as certain Butler groups. The following corollary is due to Arnold and Dugas [5]; an alternate proof is given in [28].

Corollary 4.6. Suppose $\mathbf{F}=\left(F, F_{0}, F_{1}\right) \in \operatorname{Rep}_{2} R$ has finite rank and $F_{0}, F_{1}$ are pure in $F$. Then $\mathbf{F}$ is a direct sum of $R_{2}$-modules of ranks 1 and 2.

We also observe that our decomposition theorem enables one to turn decomposable $R_{2}$-modules into indecomposable, complemented $R_{4}$-modules:

Corollary 4.7. Suppose $\mathbf{F}=\left(F, F_{0}, F_{1}\right) \in$ Rep $_{2} R$ has finite rank and $F_{0}, F_{1}$ are pure in $F$. If $\mathbf{F}$ has no rank 1 summands, then there exist pure submodules $F_{2}, F_{3} \subseteq$ 
$F$ such that $\left(F, F_{0}, F_{2}, F_{1}, F_{3}\right)$ is a complemented, indecomposable representation in $\operatorname{Rep}_{4} R$.

Additional consequences of the main decomposition theorem will be given in the sections to come, in both the global and local cases (Sections 2 and 3, respectively). We remark that the existence of indecomposable, complemented $R_{3}$-modules of infinite rank was, for a while, a matter of considerable doubt. These objects indeed exist, and we detail their construction in Section 4 The heart of the matter is Proposition 4.3, an old result in abelian group theory due to Reid [47.

In Section 5, we apply our results to Butler groups. Despite the fact that Butler groups are a special class of torsion-free abelian groups, they turn out to be as complex as the totality of torsion-free abelian groups [21, 30, 31. Every ring with free additive group is realizable as the endomorphism ring of many Butler groups from a proper class, for instance; see 21. Hence we will place a number of restrictions on the Butler groups we study, most notably restrictions on their ranks and typesets. (Recall that a group of finite rank is a Butler group if it is a pure subgroup of a completely decomposable torsion-free group of finite rank; equivalent conditions are discussed in the forthcoming book [44].) Butler groups with a critical typeset of the form $T_{n}$ (an antichain of length $n$ with a bottom element) for $n=2$ are classified in [43] as direct sums of indecomposable $T_{2}$-Butler groups of rank $\leq 2$. This also follows from $R e p_{2} R$-considerations in [5, 28. We will bring our machinery in $R e p_{n} R$ for $n=2,3$ and 4 to bear on $T_{n}$-groups in the final section, obtaining group-theoretical results about Butler groups of various torsion-free ranks, both finite and infinite.

\section{The Global Case of the Main Theorem}

Throughout this section $R$ will denote any principal ideal domain that is not a field. If $M$ is an $R$-module, $p \in R$ and $e \in M \backslash p M$, we say $e$ is $p$-pure in $M$. If $e$ is $p$-pure for all primes $p$ of $R$, or equivalently for every non-unit $p$ of $R$, then $e$ is said to be pure in $R$. We will constantly use the fact that pure elements $e$ of free $R$-modules $M$ are basic, i.e. $M=e R \oplus M^{\prime}$.

We will work with special objects of $R e p_{n} R$ described by the following:

Definition 2.1. A representation $\mathbf{F}=\left(F, F_{i}: i<n\right)$ with $F$ free is a complemented representation if $F_{0}+F_{1}=F, F_{i}$ is pure in $F$ for all $i$, and $F_{i} \cap F_{j}=0$ for all $i \neq j$.

Lemma 2.2. Suppose $\mathbf{F}=\left(F, F_{0}, F_{1}, F_{2}\right) \in \operatorname{Rep}_{3} R$ is such that $F=F_{0} \oplus F_{1}$. Let $\pi_{i}: F \longrightarrow F_{i}$ for $i=0,1$ be the canonical projections and assume

$F_{2} \pi_{0}=\bigoplus_{i<\lambda} R t_{i} a_{i}$, where $F_{0}=\bigoplus_{i<\lambda} R a_{i}$ and $t_{i}=t_{i}^{\prime} \cdot t_{0}$ in $R$ for all $i<\lambda$.

Choose $c_{0} \in F_{1}$ with $d_{0}=t_{0} a_{0}+c_{0} \in F_{2}$, and write $c_{0}=s_{0}$ e for some pure element $e$ of $F_{1}$. If $F_{2} \pi_{1} \subseteq s_{0} F_{1}$, then $\left(R a_{0} \oplus R e, R a_{0}, R e, R d_{0}\right)$ is a summand of F.

Proof. By hypothesis we have

$$
\begin{aligned}
F /\left(F_{1} \oplus F_{2}\right) & =\left(F_{1} \oplus F_{0}\right) /\left(F_{1} \oplus F_{2}\right) \cong F_{0} / F_{1} \pi_{0} \\
& =\bigoplus_{i<\lambda} R a_{i} / \bigoplus_{i<\lambda} R t_{i} a_{i} \cong \bigoplus_{i<\lambda}\left(R / t_{i} R\right) .
\end{aligned}
$$


Hence $t_{i} a_{i} \in F_{1} \oplus F_{2}$ and there are unique elements $c_{i} \in F_{1}, d_{i} \in F_{2}$ with $t_{i} a_{i}=$ $d_{i}-c_{i}$. Clearly $\bigoplus_{i<\lambda} R d_{i}=\bigoplus_{i<\lambda} R\left(t_{i} a_{i}+c_{i}\right) \subseteq F_{2}$, and it is easy to check

$$
F_{2}=\bigoplus_{i<\lambda} R d_{i} \text { with } d_{i}=t_{i} a_{i}+c_{i} \quad(i<\lambda) .
$$

Since $c_{0}=s_{0} e$ and $e$ is pure in the free module $F_{1}$, we get a decomposition $F_{1}=$ $R e \oplus F_{1}^{\prime}$; let $\pi: F_{1} \longrightarrow R e$ be the canonical projection. Denote $c_{i} \pi=s_{i} e$ for all $0 \neq i<\lambda$. Since $F_{2} \pi_{1} \subseteq s_{0} F_{1}, d_{i} \pi_{1} \pi=s_{i} e \in s_{0} F_{1}$, and $e$ is pure in $F_{1}, s_{0}$ must divide all $s_{i}$ for $i<\lambda$. Put $d_{0}^{\prime}=d_{0} ; s_{i}^{\prime}=\frac{s_{i}}{s_{0}}$ and $d_{i}^{\prime}=d_{i}-s_{i}^{\prime} d_{0}$ for $i>0$. Then clearly $F_{2}=R d_{0}^{\prime} \oplus F_{2}^{\prime}$, where $F_{2}^{\prime}=\bigoplus_{i>0} R d_{i}^{\prime}$ and $F_{2}^{\prime} \pi=0$. We also have $F_{0}=R a_{0} \oplus F_{0}^{\prime}$, where $F_{0}^{\prime}=\bigoplus_{0 \neq i<\lambda} R t_{i} a_{i}^{\prime}$ with $a_{i}^{\prime}=a_{i}-s_{i}^{\prime} t_{i}^{\prime} a_{0}$. Clearly $F_{2}^{\prime} \pi_{0} \subseteq F_{0}^{\prime}$, and it follows that $\mathbf{F}=\left(R a_{0} \oplus R e, R a_{0}, R e, R d_{0}\right) \oplus\left(F_{0}^{\prime} \oplus F_{1}^{\prime}, F_{0}^{\prime}, F_{1}^{\prime}, F_{2}^{\prime}\right)$, as desired.

Note that although a sum $e_{1}+e_{2}$ of two pure elements $e_{1}, e_{2}$ need not be pure, $p_{1} e_{1}+p_{2} e_{2}$ is at least $p_{i}$-pure for distinct primes $p_{i}(i=1,2)$. Except for trivial cases it will not be pure; take $e_{1}=-e_{2}$, for instance. The following trick to arrange primes was used in Cohen and Gluck's classic paper [17, and generalizes the above remark. If $\left\{s_{0}, \ldots, s_{n-1}\right\}$ is a set of elements in $R$, let $\left(s_{i}: i \in n\right)$ denote its g.c.d., which is unique up to units in $R$.

Lemma 2.3. Let $\mathcal{P}$ be a finite set of primes of $R$, and $e_{0}, \ldots, e_{n-1}$ be p-pure elements of an $R$-module $M$ for all $p \in \mathcal{P}$. Assume that $s_{0}, \ldots, s_{n-1} \in R$ are given such that $\left(s_{i}: i \in n\right)=1$. Then we can find $t_{0}, \ldots, t_{n-1} \in R$ such that $\sum_{i \in n} t_{i} s_{i} e_{i}$ is $p$-pure in $M$ for all $p \in \mathcal{P}$, and $\left(t_{i} s_{i}: i \in n\right)=1$ as well.

Proof. Enumerate $\mathcal{P}=\left\{p_{0}, \ldots, p_{m-1}\right\}$ without repetition for some $m \geq 1$. For each $i \in m$, we may choose $\varphi(i) \in n$ such that $p_{i}$ does not divide $s_{\varphi(i)}$ because $\left(s_{i}: i \in n\right)=1$. This determines a function $\varphi: m \longrightarrow n$. For $i \in n$, put $t_{i}=\prod\left\{p_{k} \in \mathcal{P}: \varphi(k) \neq i\right\}$.

Suppose $p$ divides $t_{i} s_{i}$ for all $i \in n$. Since $\left(s_{i}: i \in n\right)=1$, there is an $i \in n$ with $p \mid t_{i}$, hence $p$ has a name, say $p=p_{k}$ for some $k \in m$. If $\varphi(k)=j$, then clearly $p_{k} \nmid t_{j}$ by definition of $t_{j}$, hence $p_{k} \mid s_{j}$ since $p_{k} \mid t_{j} s_{j}$. This is impossible because $p_{k} \nmid s_{\varphi(k)}=s_{j}$ by definition of $\varphi$. The condition $\left(t_{i} s_{i}: i \in n\right)=1$ is therefore met.

Now suppose $p_{k} \in \mathcal{P}$ and $p_{k}$ divides $\sum_{i \in n} t_{i} s_{i} e_{i}$. If $\varphi(k)=j$, then $p_{k}$ also divides $t_{i}$ for all $i \neq j$, hence $p_{k}$ divides $\sum_{i \in n} t_{i} s_{i} e_{i}-\sum_{j \neq i \in n} t_{i} s_{i} e_{i}=t_{j} s_{j} e_{j}$. But since $e_{j}$ is $p_{k}$-pure, $p_{k}$ divides $t_{j} s_{j}$, and we obtain $p_{k} \mid\left(s_{i} t_{i}: i \in n\right)=1$, a contradiction.

Next we consider a fixed divisibility chain $t_{n-1}\left|t_{n-2}\right| \ldots \mid t_{0}$ in $R$. We abbreviate divisibility by $n-1|n-2| \ldots \mid 0$, and the corresponding quotients $\frac{t_{i}}{t_{k}}$ in $R$ by $i / k$ for $i<k$. The divisibility chain gives rise to a class of $(n-1) \times n$ matrices with entry $i / k \cdot *$ in the row labeled by $k$ and the column labeled by $i<k(*=$ any element of $R$ ), and an arbitrary entry $*$ for $i \geq k$. Note the the rows are labeled by $1, \ldots, n-1$, and the columns by $0, \ldots, n-1$. The matrices have the following 
form, where we suppress the $*$ in $i / k \cdot *$.

$$
\left(\begin{array}{ccccc}
0 / 1 & * & * & * & * \\
0 / 2 & 1 / 2 & * & * & * \\
0 / 3 & 1 / 3 & 2 / 3 & * & * \\
\vdots & \vdots & \vdots & \vdots & \vdots \\
0 / n-1 & 1 / n-1 & 2 / n-1 & 3 / n-1 & *
\end{array}\right)
$$

We also fix $j \in n$ and have a forbidden $j$-th column (the column labeled by $j$ ). Let $\Pi$ be any product of $n-1$ factors which are entries from exactly $n-1$ rows and $n-1$ columns, but not from the forbidden $j$-th column. We claim that $0 / j$ divides $\Pi$ in $R$.

The claim follows by an induction on $r<j$, as follows: If the columns and rows

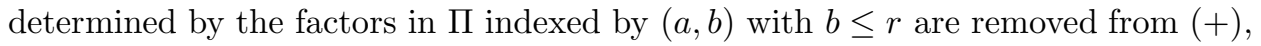
then we claim $\Pi=0 / r^{\prime} \cdot \Pi^{\prime}$ where $\Pi^{\prime}$ is the product of all factors coming from rows labeled by $(c, d)$ with $c>r^{\prime}$, where $r^{\prime}$ is the index of the largest row we have removed.

Note that if $r=0$ and we remove column 0 along with the appropriate row labeled by $\ell$, the claim is ensured by the entry $0 / \ell$.

In the induction step we remove column $r$ and some row, say $k$, according to the entry $(k, r)$ used in $\Pi$. If $k<r^{\prime}$, the claim $\Pi=0 / r^{\prime} \cdot \Pi^{\prime}$ holds trivially. The case $k=r^{\prime}$ is impossible, because row $r^{\prime}$ was removed earlier. If $k>r^{\prime}$, we must show that $\Pi=0 / k \cdot \Pi^{\prime \prime}$. Clearly the shrunken matrix has only $*$ 's on the diagonal above $k$, hence the corresponding rows have done their duty and just wait to be removed.

By hypothesis $\Pi=0 / r^{\prime} \cdot \Pi^{\prime}$ and $\Pi^{\prime}$ has a factor $r / k$ from the new entry $(k, r)$ used for $\Pi$. Since $r$ rows are removed, we have $r^{\prime} \geq r$, hence $r^{\prime} \mid r$ from our divisibility chain, and $\left(0 / r^{\prime}\right) \cdot(r / k)=(0 / k)\left(r / r^{\prime}\right)$ is a product in $R$. Now it is clear that $\Pi=(0 / k) \cdot \Pi^{\prime \prime}$ with $\Pi^{\prime \prime}$ a product that still has all factors of $\Pi$ coming from entries of rows after $k$. The induction is complete.

If $k \geq j$, the claim will follow, and this must happen after at most $j-1$ steps, which saves us from case distinction due to the forbidden column $j$.

Corollary 2.4. If $t_{n-1}\left|t_{n-2}\right| \ldots \mid t_{0}$ is a given divisibility chain in $R$ and if $\left|\Omega_{j}\right|$ denotes the determinant of the $(n-1) \times(n-1)$ matrix $\boxplus$ above after removing its $j$-th column for some $j<n$, then $\frac{t_{0}}{t_{j}}$ divides $\left|\Omega_{j}\right|$ in $R$.

Proof. Recall that the determinant $\left|\Omega_{j}\right|$ is a sum of all products of the factors considered in the discussion above. Each of them is divisible by $\frac{t_{0}}{t_{j}}$, hence $\left|\Omega_{j}\right|$ is, and the corollary follows.

Remark. We will need only a special case of Corollary 2.4, where entries labeled by $(k, j)$ of the matrices (田) are 0 for all $k>j$. In this case the corollary can also be deduced by means of row-by-row Laplace developments, beginning with the top row of (田).

Corollary 2.4 is used to obtain

Proposition 2.5. Let $t_{n-1}\left|t_{n-2}\right| \ldots \mid t_{0}$ be a given divisibility chain in $R$, and $d_{0 k} \in$ $R(k \in n)$ be given such that $\left(d_{0 k} \frac{t_{0}}{t_{k}}: k \in n\right)=1$. Then it is possible to choose additional elements $d_{i j} \in R \quad(1 \leq i<n, j \in n)$ such that the $n \times n$ matrix 
$\Delta=\left(s_{i j}\right)_{i, j \in n}$ defined by

$$
s_{i j}= \begin{cases}d_{i j} & \text { if } \quad i \leq j \\ d_{i j} \frac{t_{j}}{t_{i}} & \text { if } \quad i>j\end{cases}
$$

has determinant $|\Delta|=1$.

Proof. Let $\Delta_{i j}$ denote the minors of $\Delta$ obtained by removing the row of $\Delta$ indexed by $i$ and the column indexed by $j$. By induction on $n$, we may assume $d_{i j} \in R$ $(i, j \in n-1)$ have been chosen so that $\left|\Delta_{n-1, n-1}\right|=\left(d_{0 k} \frac{t_{0}}{t_{k}}: k \in n-1\right)$. [Note that we also could assume $d_{i j}=0$ for $i<j$ in accordance with the remark after Corollary 2.4.] Then since $\left|\Delta_{n-1, n-1}\right|$ and $d_{0, n-1} \frac{t_{0}}{t_{n-1}}$ are coprime in $R$, we may choose $d, t \in R$ with

$$
1=d \cdot\left|\Delta_{n-1, n-1}\right|+t d_{0, n-1} \frac{t_{0}}{t_{n-1}} .
$$

If we put $d_{i, n-1}=0$ for $0<i<n$, and $d_{n-1, n-1}=(-1)^{n-1} d$, then up to sign we have

$$
|\Delta|=d \cdot\left|\Delta_{n-1, n-1}\right|+d_{0, n-1}\left|\Delta_{0, n-1}\right|
$$

by Laplace applied to the last column. If we can choose $d_{n-1, j} \in R(j \in n-1)$ so that $\left|\Delta_{0, n-1}\right|=t \frac{t_{0}}{t_{n-1}}$, then $|\Delta|$ is \pm 1 and the proof is complete. Let $\Omega_{j}$ be the matrix obtained from $\Delta$ by deleting the rows of $\Delta$ indexed by 0 and $n-1$ (the first and last rows), together with the columns of $\Delta$ indexed by $j$ and $n-1$, where $j \in n-1$. Then $\Omega_{j}$ has the form described in Corollary 2.4, hence $\left|\Omega_{j}\right|=\frac{t_{0}}{t_{j}} \cdot r_{j}$, where $r_{j} \in R$. We now carry out Laplace expansion across the top row of $\Delta_{n-1, n-1}$ to see that

$$
\left|\Delta_{n-1, n-1}\right|=\sum_{j \in n-1}(-1)^{j} d_{0 j}\left|\Delta_{j}\right|=\sum_{j \in n-1}(-1)^{j} d_{0 j} \frac{t_{0}}{t_{j}} r_{j}=\left(d_{0 k} \frac{t_{0}}{t_{k}}: k \in n\right) .
$$

Therefore $\left(r_{j}: j \in n-1\right)=1$. At the same time, we see by expanding about the bottom row of $\Delta_{0, n-1}$ that, up to sign,

$$
\left|\Delta_{0, n-1}\right|=\sum_{j \in n-1}(-1)^{j} d_{n-1, j} \frac{t_{j}}{t_{n-1}}\left|\Delta_{j}\right|=\frac{t_{0}}{t_{n-1}} \sum_{j \in n-1}(-1)^{j} d_{n-1, j} r_{j} .
$$

Then because $\left(r_{j}: j \in n-1\right)=1$, the elements $d_{n-1, j}(j \in n-1)$ can be chosen so that the last expression in brackets is \pm 1 , as desired. This completes the proof.

Theorem 2.6. Let $\mathbf{F}=\left(F, F_{0}, F_{1}, F_{2}\right) \in$ Rep $_{3} R$ be a (nonzero) complemented representation of finite rank. Then $\mathbf{F}$ is a direct sum of complemented $R_{3}$-modules of ranks 1 and 2 .

Proof. Since $F$ is finite rank free, $F_{i}$ is also free of finite rank. If $F_{2}$ has rank $r k F_{2}<r k F_{0}$, then we can write $F_{2}=\bigoplus_{i \in n} R\left(a_{i}+b_{i}\right)$ with $a_{i} \in F_{0}$ and $b_{i} \in F_{1}$, where $\left\langle a_{i}: i \in n\right\rangle$ is contained in a direct summand $F_{0}^{\prime}$ of $F_{0}$ of rank $\leq r k F_{2}$. Then $F_{0}=F_{0}^{\prime} \oplus C$ and $\mathbf{F}=(C, C, 0,0) \oplus\left(F_{0}^{\prime} \oplus F_{1}, F_{0}^{\prime}, F_{1}, F_{2}\right)$. Since $(C, C, 0,0) \neq 0$ decomposes into rank 1 summands and $r k F_{2}=r k F_{0}^{\prime}$, we may assume $r k F_{2} \geq$ $r k F_{0}$ (and by symmetry $r k F_{2} \geq r k F_{1}$ ) for our original $R_{3}$-module $\mathbf{F}$. Then since $F_{2} \oplus F_{i} \subseteq F_{0} \oplus F_{1}$, also $r k F_{2} \leq r k F_{i}$ for $i=0,1$, hence $r k F_{0}=r k F_{1}=r k F_{2}=n$ for some $n$.

We now show that our (adjusted) representation $\mathbf{F}$ can be brought into the special form required by Lemma 2.2. To that end, let $\pi_{i}: F \longrightarrow F_{i}(i=0,1)$ be 
the projections relative to $F=F_{0} \oplus F_{1}$. Clearly $F_{0} \supseteq F_{2} \pi_{0}$ is finitely generated, and Gauss' theorem applies. We get stacked bases for $F_{0}$ and this submodule, namely

$$
\left\{\begin{array}{l}
F_{0}=\bigoplus_{i \in n} R a_{i} \\
\text { There is a divisibility chain } t_{n-1}\left|t_{n-2}\right| \ldots \mid t_{0} \text { such that } \\
F_{2} \pi_{0}=\bigoplus_{i \in n} R t_{i} a_{i}
\end{array}\right.
$$

and similarly for $F_{1} \supset F_{2} \pi_{1}$ we obtain

$$
\left\{\begin{array}{l}
F_{1}=\bigoplus_{i \in n} R b_{i} \\
\text { There is a divisibility chain } s_{0}\left|s_{1}\right| \ldots \mid s_{n-1} \text { such that } \\
F_{2} \pi_{1}=\bigoplus_{i \in n} R s_{i} b_{i} .
\end{array}\right.
$$

Note that none of the $s_{i}, t_{i}$ are 0 , and divisibility is expressed in opposite orders in (11) and (2). Since $s_{0} \mid s_{i}$ for all $i \in n$ we have $F_{2} \pi_{1} \subseteq s_{0} F_{1}$, a condition required in Lemma 2.2

As in the proof of Lemma 2.2 we may choose unique $c_{i} \in F_{1}$ such that $d_{i}=$ $t_{i} a_{i}+c_{i}$ and $F_{2}=\bigoplus_{i \in n} R d_{i}$. Write $c_{i}=g_{i} e_{i}$ with $g_{i} \in R$ and $c_{i}$ pure in $F_{1}$ for all $i<n$. Because $F_{2} \pi_{1}$ has rank $n$, the sum

$$
F_{2} \pi_{1}=\bigoplus_{i \in n} R g_{i} e_{i}=\bigoplus_{i \in n} R s_{i} b_{i} \subseteq s_{0} F_{1}
$$

is indeed direct. Since $e_{i}$ is pure in $F_{1}, s_{0}$ must divide $\left(g_{i}: i \in n\right)$, and since $b_{0}$ is pure in $F_{1}$, the converse holds as well. Therefore $s_{0}=\left(g_{i}: i \in n\right)$. Clearly, $d_{0}$ is pure in $F_{2}$, which is pure in $F$, hence $d_{0}$ is pure in $F$ as well. The element $d_{0}=t_{0} a_{0}+g_{0} e_{0}$ must satisfy $\left(t_{0}, g_{0}\right)=1$. We deduce $\left(\frac{g_{i} t_{0}}{s_{0} t_{i}}: i \in n\right)=1$.

If $\mathcal{P}$ denotes all primes dividing $g_{n}$, then by Lemma 2.3 , there are elements $d_{0 i} \in R(i \in n)$ such that

$$
e=\sum_{i \in n} d_{0 i}\left(\frac{g_{i} t_{0}}{s_{0} t_{i}}\right) e_{i}
$$

is $p$-pure in $F_{1}$ for all $p \in \mathcal{P}$, and such that

$$
\left(d_{0 i} \frac{g_{i} t_{0}}{s_{0} t_{i}}: i<n\right)=1 .
$$

It follows that $s_{0} e=\sum_{i \in n} d_{0 i} \frac{t_{0}}{t_{i}} c_{i}$ is pure in $\bigoplus_{i \in n} R c_{i}$, hence $s_{0} e$ is $p$-pure in $F_{1}$ for all primes $p \notin \mathcal{P}$ because $F_{1} / F_{2} \pi_{2}$ is $p$-torsion-free for these primes by (2). Therefore, $e$ is pure in $F_{1}$. Because of (4) we may apply Proposition 2.5 and find an $n \times n$ matrix $\Delta$, with $|\Delta|=1$, of the form $\Delta=\left(s_{i j}\right)$ with $s_{i j}=d_{i j} \frac{t_{i}}{t_{j}}$ for $i>j$, where $d_{0 i}, t_{i}$ are given above. Since $|\Delta|=1, \Delta$ represents an automorphism of the free module $F_{2}=\bigoplus_{i \in n} R d_{i}$.

We denote $\Delta d_{i}=d_{i}^{\prime}=\sum_{j \in n} s_{i j} d_{j}$ for $i \in n$. Using $d_{i}=t_{i} a_{i}+c_{i}$ we get $d_{i}^{\prime}=$ $t_{i} a_{i}^{\prime}+c_{i}^{\prime}$, where similarly we denote $a_{i}^{\prime}=\sum_{j \in n} s_{i j} a_{j}$ and $c_{i}^{\prime}=\sum_{j \in n} s_{i j} c_{j}$. In particular, $d_{0}^{\prime}=t_{0} a_{0}^{\prime}+s_{0} e$ because of (3). Clearly $a_{i}^{\prime} \in F_{0}$, and $F_{0}=\bigoplus_{i \in n} R a_{i}=\bigoplus_{i \in n} R a_{i}^{\prime}$ since $|\Delta|=1$. Note $F_{2}^{\prime}:=\bigoplus_{i \in n} R d_{i}^{\prime} \subseteq F_{2}$. In order to show equality, we observe that $F_{0} / F_{2} \pi_{0} \cong\left(F_{0} / F_{2}^{\prime} \pi_{0}\right) /\left(F_{2} \pi_{0} / F_{2}^{\prime} \pi_{0}\right)$, hence $F_{2} \pi_{0}=F_{2}^{\prime} \pi_{0}$ because $F_{0} / F_{2} \pi_{0} \cong$ 
$\bigoplus_{i \in n} R / R t_{i} \cong F_{0} / F_{2}^{\prime} \pi_{0}$ is finitely generated. Now $F_{0}=\bigoplus_{i \in n} R a_{i}^{\prime} \supset F_{2}=\bigoplus_{i \in n} R d_{i}^{\prime}$ is of the form required by Lemma 2.2 to split off the rank 2 summand $\left(R a_{0}^{\prime} \oplus\right.$ $R e, R a_{0}^{\prime}, R e, R d_{0}^{\prime}$ ) from $\mathbf{F}$. An induction on the rank of $\mathbf{F}$ now completes the proof of the theorem.

Remark 2.7. The nonzero indecomposable, complemented $R$-representations $\left(F, F_{0}\right.$, $\left.F_{1}, F_{2}\right)$ of finite rank have the following forms:

(1) $(R, R, 0,0)$ and $(R, 0, R, 0)$;

(2) $(R e \oplus R f, R e, R f, R(a e+b f))$, where $a$ and $b$ are nonzero and coprime in $R$.

\section{The Local Case of the Main Theorem}

In this section, we prove our main theorem in the much easier case where the base ring $R$ is a discrete valuation domain. Let $p R$ be the maximal ideal of $R$, and let $\mathbf{F}=\left(F, F_{i}: i<3\right) \in \operatorname{Rep}_{3} R$ be a nonzero, complemented representation of finite rank. We claim that $\mathbf{F}$ has a direct summand of rank 1 or 2 . In what follows, $\pi_{i}: F=F_{0} \oplus F_{1} \rightarrow F_{i}(i=0,1)$ denotes projection.

We first show that if $F /\left(F_{1}+F_{2}\right)$ is not torsion, then $\mathbf{F}$ has a rank 1 direct summand. An easy check verifies $F /\left(F_{1}+F_{2}\right)=\left(F_{0} \oplus F_{1}\right) /\left(F_{1}+F_{2}\right) \cong F_{0} / F_{2} \pi_{0}$. By assumption, the (finitely generated) quotient $F /\left(F_{1}+F_{2}\right)$ has a direct summand isomorphic to $R$. Therefore we obtain a decomposition $F=F_{0}^{\prime} \oplus F_{0}^{\prime \prime}$ with the first summand isomorphic to $R$ and $F_{2} \pi_{0} \subseteq F_{0}^{\prime \prime}$. Since clearly $F_{2} \subseteq F_{2} \pi_{0} \oplus F_{1}$, it follows that $\mathbf{F}=\left(F_{0}^{\prime}, F_{0}^{\prime}, 0,0\right) \oplus\left(F_{0}^{\prime \prime} \oplus F_{1}, F_{0}^{\prime \prime}, F_{1}, F_{2}\right)$, as desired.

Now we assume that $F_{0} / F_{2} \pi_{0}$ is torsion. Since $F_{2}$ is pure in $F$, it follows that $F_{0} \neq 0$. By Gauss' theorem, we get stacked bases $F_{0}=\bigoplus_{1 \leq i \leq m} R e_{i}$ and $F_{2} \pi_{0}=$ $\bigoplus R p^{n_{i}} e_{i}$, where $n_{1} \geq \cdots \geq n_{m} \geq 0$. Since $F_{2} \cap F_{1}=0$, there are unique $1 \leq i \leq m$

$f_{i} \in F_{2}$ such that $f_{i} \pi_{0}=p^{n_{i}} e_{i}$ for $i \leq i \leq m$. It is easy to check that the $f_{i}$ 's are independent and span $F_{2}$, whence $F_{2}=\bigoplus_{1 \leq i \leq m} R f_{i}$. Since $F_{2}$ is $p$-pure in $F$ and $F_{2} \cap F_{0}=0, f_{1} \pi_{1}$ is a nonzero, pure element of $F_{1}$ if $n_{1}>0$. If $n_{1}=0$, we may relabel to put ourselves in the situation where the $p$-height $h\left(f_{1} \pi_{1}\right)$ of the nonzero element $f_{1} \pi_{1}$ is minimal in the set $\left\{h\left(f_{i} \pi_{1}\right): 1 \leq i \leq m\right\}$. Let $R g$ be the purification of $R f_{1} \pi_{1}$ in $F_{1}$. Then $F_{1}=R g \oplus F_{1}^{\prime}$; for each $i$, write $r_{i} g\left(r_{i} \in R\right)$ for the projection of $f_{i} \pi_{1}$ onto $R g$. Then by construction $r_{i} / r_{1} \in R$ for all $i$. Put $f_{i}^{\prime}=f_{i}-\left(r_{i} / r_{1}\right) f_{1}$ and $e_{i}^{\prime}=e_{1}-p^{n_{1}-n_{i}}\left(r_{i} / r_{1}\right) e_{i}$ for $1<i \leq m$. Clearly $F_{0}=R e_{0} \oplus F_{0}^{\prime}$ and $F_{2}=R f_{1} \oplus F_{2}^{\prime}$, where $F_{0}^{\prime}=\underset{1<i \leq m}{\bigoplus} R e_{i}^{\prime}$ and $F_{2}^{\prime}=\bigoplus_{1<i \leq m} R f_{i}^{\prime}$. Observe $F_{2}^{\prime} \pi_{0}=\bigoplus_{1<i \leq m} R p^{n_{i}} e_{i}^{\prime} \subseteq F_{0}^{\prime}$, and $F_{2}^{\prime} \pi_{1} \subseteq F_{1}^{\prime}$ because we have arranged by our choice of bases that $F_{2}^{\prime} \pi_{1}$ has trivial projection onto $R g$. Thus $F_{2}^{\prime} \subseteq F_{0}^{\prime} \oplus F_{1}^{\prime}$. Evidently $\mathbf{F}=\left(R e_{0} \oplus R g, R e_{0}, R g, R f_{1}\right) \oplus\left(F_{0}^{\prime} \oplus F_{1}^{\prime}, F_{0}^{\prime}, F_{1}^{\prime}, F_{2}^{\prime}\right)$, as desired.

An induction on the rank of $\mathbf{F}$ now completes the proof of the decomposition theorem:

$\mathbf{F}$ is a direct sum of (complemented) indecomposable representations of ranks 1 and 2.

Remark 3.1. Up to isomorphism, the indecomposable, complemented representations $\neq 0$ in $\operatorname{Rep}_{3} R$ have the following forms:

(1) $(R, R, 0,0)$ and $(R, 0, R, 0)$;

(2) $\left(R e \oplus R f, R e, R f, R\left(p^{n} e+p^{m} f\right)\right)$, where $0 \in\{n, m\}$. 
We conclude with some remarks about various subcategories of $\operatorname{Rep}_{3} R$.

For $0 \leq i \leq j$, let $C_{3}(i, j)$ denote the category of finite-rank objects

$\left(F, F_{i}: i<3\right)$ subject to the following conditions:

(i) $F_{i}$ is pure in $F$ for $i<3$;

(ii) $F_{i} \cap F_{j}=0$ if $i \neq j$;

(iii) the torsion parts of the factors $F /\left(F_{0} \oplus F_{1}\right)$ and $F /\left(F_{1} \oplus F_{2}\right)$ are bounded by $p^{j}$; and

(iv) at least one of the factors in (iii) is bounded by $p^{i}$.

We are thankful to D. Arnold for discussions about the representation types of $C_{3}(i, j)$ for different values of the parameters. If $1 \leq i<j$, the category $C_{3}(i, j)$ contains indecomposables of arbitrarily large rank, hence has infinite representation type. The category $C_{3}(i, j)$ has so-called "endo-wild" representation type if $2 \leq i \leq j$. The latter notion is a generalization of wild representation type for representations of posets over fields, and indicates that a meaningful classification of all indecomposables in $C_{3}(i, j)$ is unfeasible when $2 \leq i \leq j$.

Note that after a permutation of the distinguished submodules, each representation in $C_{3}(0, j)$ is a finite-rank, complemented representation over $R$. Conversely, every finite-rank complemented representation in $\operatorname{Rep}_{3} R$ lies in $C_{3}(0, j)$ for some value of $j$. Remark 3.1 can be used to deduce that $C_{3}(0, j)$ contains exactly $3 j+4$ nonzero indecomposables, hence is of finite representation type.

Finally, it is relatively straightforward to verify that the following representation $\left(F, F_{i}: i<3\right)$ of rank $2 m$ is an indecomposable member of $C_{3}(1, j)$ if $j \geq 1$.

Let $e_{i j}(1 \leq i \leq m, j=0,1)$ be a set of generators for a free $R$-module of rank $2 m$, and define:

$$
\begin{aligned}
& F_{j}=\bigoplus_{1 \leq i \leq m} R p e_{i j} \text { for } j=0,1 ; \\
& F=F_{0} \oplus F_{1}+\underset{1 \leq i \leq m}{\bigoplus} R\left(e_{i-1,0}+e_{i 1}\right), \text { where } e_{00}=0 ; \\
& F_{2}=\bigoplus_{1 \leq i \leq m} R p\left(e_{i 0}+e_{i 1}\right) .
\end{aligned}
$$

\section{Applications of the Main Theorem}

In this section, we show how Theorem 2.6 can be applied to obtain new facts about modules with two distinguished submodules.

Let $\Sigma_{2} R$ denote the full subcategory of $R e p_{2} R$ consisting of all finite-rank representations $\left(F, F_{0}, F_{1}\right)$ such that $F=F_{0}+F_{1}$ (we make no assumption about the purity of the submodules here). We thank D. Arnold for pointing out the following category equivalence.

Proposition 4.1. There is an additive equivalence from $\Sigma_{2} R$ onto the category of all finite-rank, complemented representations in Rep $R$.

We briefly indicate the (covariant) functors involved in the proof of this result. Define

$$
f: \Sigma_{2} R \longrightarrow \operatorname{Rep}_{3} R
$$

by putting

$$
f(\mathbf{F})=\left(F_{0} \times F_{1}, F_{0}, F_{1}, F_{2}\right),
$$

where $\mathbf{F}=\left(F, F_{0}, F_{1}\right) \in \Sigma_{2} R$ and $F_{2}$ is the kernel of the homomorphism $(x, y) \mapsto$ $x+y$ from the external direct sum $F_{0} \times F_{1}$ onto $F$. 
It is straightforward to verify that $f(\mathbf{F})$ is complemented, and that $f$ commutes with direct sums in the category.

Conversely, if $\mathbf{U}=\left(U, U_{0}, U_{1}, U_{2}\right) \in \operatorname{Rep}_{3} R$ is complemented and of finite rank, then it is easy to verify that

$$
\left.g(\mathbf{U})=\left(U / U_{2},\left(U_{0} \oplus U_{2}\right) / U_{2},\left(U_{1} \oplus U_{2}\right) / U_{2}\right)\right)
$$

lies in $\Sigma_{2} R$. Morphisms $\mathbf{F} \longrightarrow \mathbf{F}^{\prime}$ induce morphisms $f(\mathbf{F}) \longrightarrow f\left(\mathbf{F}^{\prime}\right)$ in a natural way, and similarly for the other functor $g$.

Observe that if $\left(F, F_{0}, F_{1}\right) \in \Sigma_{2} R$, then

$$
g f\left(F, F_{0}, F_{1}\right)=\left(\left(F_{0} \times F_{1}\right) / F_{2},\left(F_{0} \oplus F_{2}\right) / F_{2},\left(F_{1} \oplus F_{2}\right) / F_{2}\right) \cong\left(F, F_{0}, F_{1}\right)
$$

because the canonical isomorphism

$$
\left(F_{0} \times F_{1}\right) / F_{2} \longrightarrow F\left((x, y)+F_{2} \mapsto x+y\right)
$$

carries the submodule $\left(F_{0} \oplus F_{2}\right) / F_{2}$ onto $F_{0}$ and $\left(F_{1} \oplus F_{2}\right) / F_{2}$ onto $F_{1}$. Thus $g f=1_{\Sigma_{2} R}$. A similar check reveals that $f g$ is the other identity.

In view of Remark 2.7 and the discussion above, we have the following classification of objects in $\Sigma_{2} R$ :

Corollary 4.2. Nonzero objects in $\Sigma_{2} R$ are direct sums of indecomposables of rank $\leq 2$. Up to isomorphism, the indecomposable objects $\neq 0$ in $\Sigma_{2} R$ have the following forms: $(R, R, 0),(R, 0, R)$ and $(R e, R a e, R b e)$, where $a$ and $b$ are nonzero and coprime in $R$.

It seems rather hard to visualize what kinds of techniques in $R e p_{2} R$ itself (opposed to $\operatorname{Rep}_{3} R$ ) would be suitable to obtain the decompositions of objects $\left(F_{0}+\right.$ $\left.F_{1}, F_{0}, F_{1}\right)$ guaranteed by Corollary 4.2 . On the other hand, there seem to be no methods available in $\operatorname{Rep}_{3} R$ to produce even a single specimen of an indecomposable, complemented representation of infinite rank.

Concerning this last problem, we remark that it is easy to verify (and we will do so below) that if $\mathbf{F}=\left(F, F_{i}: i<3\right)$ is complemented and $F / F_{2}$ is indecomposable, then $\mathbf{F}$ is indecomposable. The problem here lies in arranging somehow that $F_{0} \cap$ $F_{2}=0$ and $F_{1} \cap F_{2}=0$. Let us concentrate on the case $R=\mathbb{Z}$. Working in the spirit of Corollary 4.2 and using the functors $f$ and $g$ described above, we are able to formulate the problem at hand as one about abelian groups with two special subgroups:

Which (indecomposable) abelian groups can be written as sums of two free subgroups?

This fundamental question was answered some time ago by Reid [47]. We supply our own proof.

Proposition 4.3. (i) If $G$ is a torsion-free abelian group of finite torsion-free rank which is a sum of two free subgroups, then $G$ is finitely generated and free.

(ii) If $G$ is an abelian group of infinite torsion-free rank not less than its cardinality (in particular, any torsion-free group of infinite rank), then $G$ is the sum of two free subgroups.

Proof. (i) If $G=F_{0}+F_{1}$ for some free subgroups $F_{i}$, then $F_{i}$ must have finite rank and consequently must be finitely generated by assumption on the rank of $G$. Hence $G$ is finitely generated and $G=E \oplus F$ for some finite group $E$ and some finitely generated free group $F$. Now it is clear that $E=0$ and (i) follows. 
(ii) Let $\kappa \geq \omega$ be the torsion-free rank of $G$, and let $F_{0}=\bigoplus_{i<\kappa} \mathbb{Z} x_{i}$ be a maximal free subgroup of $G$. Then we may enumerate the nonzero elements of $G / F_{0}$ as $\left\{g_{j}+F_{0}: j<\kappa\right\}$. For each $j$, there is a least positive integer $n_{j}$ with $n_{j} g_{j} \in F_{0}$; let $\left[n_{j} g_{j}\right]$ denote its support with respect to the given basis for $F_{0}$. Observe that for each $i<\kappa$, the set $\bigcup_{j \leq i}\left[n_{j} g_{j}\right]$ has cardinality $<\kappa$. Hence we can define (by recursion) a function $\tau: \kappa \longrightarrow \kappa$ such that for each $i<\kappa$,

$$
\bigcup_{j \leq i}\left[n_{j} g_{j}\right] \cap\{\tau(j): j \leq i\}=\emptyset .
$$

Put $F_{1}=\sum_{i<\kappa} \mathbb{Z}\left(g_{i}+x_{\tau(i)}\right) \subset G$. Clearly, $G=F_{0}+F_{1}$. Moreover, it follows by our definition of $\tau$ that $F_{1}=\bigoplus_{i<\kappa} \mathbb{Z}\left(g_{i}+x_{\tau(i)}\right)$, hence $F_{1}$ is free.

Corollary 4.4. Let $G$ be an indecomposable, torsion-free group of infinite rank $\kappa$. Let $F_{i}(i=0,1)$ be free subgroups of $G$ whose sum is $G$, and let $F_{2}$ be the kernel of the map

$$
F_{0} \times F_{1} \longrightarrow F((x, y) \mapsto x+y) .
$$

Then $\mathbf{F}=\left(F_{0} \times F_{1}, F_{0}, F_{1}, F_{2}\right) \in R_{\text {Rep }} \mathbb{Z}$ is an indecomposable, complemented representation of rank $\kappa$. If $G$ has endomorphism ring $\mathbb{Z}$, then so does $\mathbf{F}$.

Proof. Assume $G=F_{0}+F_{1}$ is indecomposable and torsion-free. Note $F / F_{2} \cong G$ is torsion-free, hence $F_{2}$ is pure in $F$. It is easy to check $F_{2} \cap F_{i}=0$ for $i=0,1$. Hence $\mathbf{F}$ is a complemented representation of rank $\kappa$. Suppose $\sigma^{2}=\sigma \in \operatorname{End}(\mathbf{F})$. Then $\sigma$ induces an idempotent in $\operatorname{End}\left(F / F_{2}\right)$, with $F / F_{2}$ indecomposable, hence by replacing $\sigma$ by $1-\sigma$ if necessary we may assume that $\sigma$ induces the zero map on $F / F_{2}$, that is, $F \sigma \subseteq F_{2}$. Then $\left(F_{i}\right) \sigma \subseteq F_{i} \cap F_{2}=0$ for $i=0,1$, whence $\sigma=0$ and $\mathbf{F}$ is indecomposable. If in addition $G$ has endomorphism ring $\mathbb{Z}$, then given any endomorphism $\sigma$ of $\mathbf{F}$ we obtain an integer $z$ so that $\sigma-z$ is zero on $F / F_{2}$, and proceed in the same way to conclude $\sigma-z=0$, whence End $\mathbf{F} \subseteq \mathbb{Z}$.

Of course, a similar strategy will work to produce indecomposables as in Corollary 4.4 over many domains besides $\mathbb{Z}$. We chose $\mathbb{Z}$ because Proposition 4.3 seems to be a noteworthy result for abelian groups, and because Corollary 4.4 will serve as a tool for constructing special Butler groups in the final section.

We turn now to a different application of Theorem 2.6. Here, we are interested in generating indecomposables in $R e p_{4} R$ from objects in $\operatorname{Rep}_{3} R$ and $R e p_{2} R$. In the setting of representations $\mathbf{U}=\left(U, U_{i}: i<n\right)$ over a field $K$, Brenner [12] realizes the trivial algebra $K$ as End $\mathbf{U}$ using four subspaces $U_{i}(i=0,1,2,3)$, and in fact provides a complete list of all such representations with $\operatorname{dim} U$ finite. An inspection of her list shows that when $\operatorname{dim} U=2 m>2, U$ and its first three subspaces constitute a finite rank complemented representation in $\operatorname{Rep}_{3} K$. Moreover, $U_{2}$ is the usual "diagonal" subspace of dimension $m$. The fourth, more "complicated" subspace $U_{3}$ has dimension $m+1$, hence $U_{i} \cap U_{3}$ must be nontrivial for all $i<4$. Hence $\mathbf{U}$ is not a complemented representation of $\operatorname{Rep}_{4} K$. In fact, the rigid representations of infinite rank given in Göbel, May [33] fail to be complemented for the same reason.

In the next section we will want to work only with complemented representations in order to apply our results to $T_{n}$-Butler groups, a setting in which each pair of distinguished submodules is required to have zero intersection. Brenner's results indicate that we cannot hope to transform a complemented representation 
as in Theorem [2.6 into a rigid one by adjoining a fourth (pure) submodule which intersects the others trivially. However, there is enough leeway to produce an indecomposable representation in this fashion. The following corollary of Theorem [2.6] will be used in Section 5 to construct Butler groups in a very special way.

Corollary 4.5. Let $R$ be a PID with infinitely many primes. Assume that $\mathbf{F}=$ $\left(F, F_{i}: i<3\right) \in \operatorname{Rep}_{3} R$ is a complemented representation of finite rank which has no direct summand of rank 1 . Then there is a pure submodule $F_{3} \subseteq F$ (there are in fact infinitely many) such that the following holds:

$\mathbf{F}^{\prime}=\left(F, F_{i}: i<4\right)$ is a complemented, indecomposable $R_{4}$-module.

Proof. Since $\mathbf{F}$ has no cyclic summands, Theorem 2.6 and Remark 2.7 provide us with the following explicit bases for the first three submodules:

$$
F_{0}=\bigoplus_{i=1}^{n} R e_{i}, F_{1}=\bigoplus_{i=1}^{n} R f_{i} \text { and } F_{2}=\bigoplus_{i=1}^{n} R\left(a_{i} e_{i}+b_{i} f_{i}\right),
$$

where $a_{i}, b_{i} \in R \backslash\{0\}$ and $\left(a_{i}, b_{i}\right)=1$ for all $i$. Denote $a_{i} e_{i}+b_{i} f_{i}$ by $d_{i}$. Since $R$ has infinitely many primes, we may choose a prime $p \in R$ which does not divide any of the coefficients $a_{i}$ and $b_{i}$. We define $F_{3} \subseteq F$ by

$$
F_{3}=\bigoplus_{i=1}^{n-1} R\left(a_{i} e_{i}+p^{i+1} b_{i+1} f_{i+1}\right) \oplus R\left(a_{n} e_{n}+p b_{1} f_{1}\right)
$$

and put $\mathbf{F}^{\prime}=\left(F, F_{i}: i<4\right) \in \operatorname{Rep}_{4} R$.

Denote the indicated basis elements of $F_{3}$ by $g_{1}, \ldots, g_{n}$ respectively. Since $\left(a_{i}, p b_{i}\right)$ $=1$ for all $i$, it follows easily that $F_{3}$ is a pure submodule of $F$, and obviously $F_{3} \cap F_{i}=0$ for $i=0,1$.

Suppose $x=\sum_{i=1}^{n} r_{i} d_{i}=\sum_{i=1}^{n} s_{i} g_{i} \in F_{3} \cap F_{2}$ is a pure element, where $r_{i}, s_{i} \in$ $R$. Upon writing these sums in terms of basis elements $e_{i}$ and $f_{i}$, and equating coefficients, we obtain $r_{1}=p s_{n}$ and $r_{i}=p^{i} s_{i-1}$ for $i>1$, hence $x \in p\left(F_{3} \cap F_{2}\right)$ and $x=0$ by purity. Therefore $F_{3} \cap F_{2}=0$ as well, and $\mathbf{F}^{\prime}$ is a complemented representation.

We now consider any $\sigma \in$ End $\mathbf{F}^{\prime}$. Since $F_{i} \sigma \subseteq F_{i}(i=0,1)$ we may write

$$
e_{i} \sigma=\sum_{k=1}^{n} r_{k}^{i} e_{k} \text { and } f_{i} \sigma=\sum_{k=1}^{n} s_{k}^{i} f_{k}
$$

for $k \leq i \leq n$, where $r_{k}^{i}, s_{k}^{i} \in R$. Since $F_{2} \sigma \subseteq F_{2}$, we may also write $d_{i} \sigma=$ $a_{i}\left(e_{i} \sigma\right)+b_{i}\left(f_{i} \sigma\right)=\sum_{k=1}^{n} t_{k}^{i}\left(a_{k} e_{k}+b_{k} f_{k}\right)$ for all $i$, where $t_{k}^{i} \in R$. Inserting the expressions (i) and equating coefficients, we obtain

$$
a_{i} r_{k}^{i}=a_{k} t_{k}^{i} \text { and } b_{i} s_{k}^{i}=b_{k} t_{k}^{i}(1 \leq i, k \leq n) .
$$

Set $k=i$ to see

$$
r_{i}^{i}=s_{i}^{i}(1 \leq i \leq n) .
$$

At this point we assume that $\sigma$ is an idempotent. If we can show that $\sigma=0$ or $\sigma=1$ then $\mathbf{F}^{\prime}$ is indecomposable and (4.5) holds. 
Using (i) and Fubini's theorem we compute

$$
e_{i} \sigma=\sum_{k=1}^{n} r_{k}^{i} e_{k}=e_{i} \sigma^{2}=\sum_{k=1}^{n}\left(\sum_{j=1}^{n} r_{k}^{j} r_{j}^{i}\right) e_{k}
$$

for all $i$, whence

$$
r_{j}^{i}=\sum_{k=1}^{n} r_{k}^{i} r_{j}^{k}(1 \leq i, j \leq n) .
$$

Now we employ the fact that $F_{3}$ is invariant under $\sigma$. For each $i$ there are elements $v_{k}^{i} \in R$ such that

$$
q_{i} \sigma=\sum_{k=1}^{n} v_{k}^{i} g_{k}
$$

Fix $i<n$ and write the $g$ 's in (v) in terms of the $e$ 's and f's, apply (i) to the left-hand side of $(\mathrm{v})$ and equate coefficients to derive

$$
\left\{\begin{array}{l}
a_{i} r_{k}^{i}=v_{k}^{i} a_{k}(1 \leq k \leq n, 1 \leq i<n) \text { and } \\
p^{i+1} b_{i+1} s_{k}^{i+1}=v_{k-1}^{i} p^{k} b_{k}(1<k \leq n, 1 \leq i<n) .
\end{array}\right.
$$

Similarly, we set $i=n$ in (v) to deduce

$$
\left\{\begin{array}{l}
a_{n} r_{k}^{n}=v_{k}^{n} a_{k}(1 \leq k \leq n) \\
b_{1} s_{k}^{1}=v_{k-1}^{n} p^{k-1} b_{k}(1<k \leq n) \\
s_{1}^{1}=v_{n}^{n}
\end{array}\right.
$$

Recall that the elements $a_{i}$ and $b_{i}$ are prime to $p$. By (vii) we see that $s_{k}^{1} \equiv 0 \bmod p$, when $k>1$, and a shift of indices in the second equation of (vi) yields $s_{k}^{i} \equiv 0 \bmod p$, when $1<i<k \leq n$. Together with (ii), these facts give us

$$
r_{k}^{i} \equiv s_{k}^{i} \equiv 0 \bmod p, 1 \leq i<k \leq n .
$$

Now (iv) reveals that $r_{i}^{i}\left(1-r_{i}^{i}\right) \equiv 0 \bmod p$ for all $i$. Replacing $\sigma$ by $1-\sigma$ if necessary, we may assume that $r_{1}^{1} \equiv s_{1}^{1} \equiv 0 \bmod p$. By setting $k=i$ and $k=i+1$ in the first and second equations of (vi), respectively, we obtain $r_{i}^{i}=v_{i}^{i}=s_{i+1}^{i+1}$ for $1 \leq i<n$. Similarly, $r_{n}^{n}=v_{n}^{n}=s_{1}^{1}$ by (vii). Therefore by (ii), we have $r_{i}^{i} \equiv s_{i}^{i} \equiv 0$ $\bmod p$ for all $i$. Setting $i=j+1$ in (iv) and employing (viii), we deduce $r_{j}^{j+1} \equiv 0$ $\bmod p$ for $j<n$. If $i>1$, we may assume by induction that $r_{j}^{j+i-1} \equiv 0 \bmod p$ for $j \leq n+1-i$. Then once again an application of (iv) and (viii) reveals that $r_{j}^{j+i} \equiv 0 \bmod p$ for $j \leq n+i$. We have shown

$$
r_{i}^{j} \equiv 0 \bmod p, 1 \leq i, j \leq n .
$$

Now (iv) implies $r_{i}^{j} \in \bigcap_{k<\omega} p^{k} R=0$ for all $i$ and $j$. By (ii), all $s_{i}^{j}$ are zero as well. Therefore $\sigma=0$, as desired.

Last, we observe that there were infinitely many choices of the prime $p \in R$, hence of the fourth submodule $F_{3} \subseteq F$. In fact, we get an infinite family of such F's with only the zero homomorphism between distinct members.

In recent accounts of representation theory and Butler groups (see [5, 28]), $R_{2^{-}}$ modules $\left(F, F_{0}, F_{1}\right)$ with pure submodules $F_{0}$ and $F_{1}$ have played a major role. It is worth pointing out how an important theorem concerning such representations 
[5, Theorem A.1], [28, Corollary 3.3] follows with relative ease from our splitting theorem for $R_{3}$-modules.

Corollary 4.6. Suppose $\mathbf{F}=\left(F, F_{0}, F_{1}\right) \in$ Rep $_{2} R$ has finite rank and $F_{0}, F_{1}$ are pure in $F$. Then $\mathbf{F}$ is a direct sum of $R_{2}$-modules of ranks 1 and 2.

Proof. Because $F / F_{0}$ is finitely-generated and torsion free, it is free. Therefore $F_{0}$ splits in $F$, say $F=F_{0} \oplus C$. We first show how Corollary 4.6 follows when $\mathbf{F}$ satisfies the following condition:

$$
F_{0} \cap F_{1}=C \cap F_{1}=0 .
$$

Denote $\mathbf{F}^{\prime}=\left(F, F_{0}^{\prime}, F_{1}^{\prime}, F_{2}^{\prime}\right)=\left(F, F_{0}, C, F_{1}\right)$. Clearly, $\mathbf{F}$ is a complemented representation in $\operatorname{Rep}_{3} R$. Using Theorem [2.6, there exists a decomposition $F=\bigoplus_{j=1}^{n} A_{j}$ into summands $A_{j}$ of rank $\leq 2$ such that $F_{i}^{\prime}=\sum_{j=1}^{n}\left(F_{i}^{\prime} \cap A_{j}\right)$ for $i=0,1,2$. Now $\left(F, F_{0}, F_{1}\right)=\bigoplus_{j=1}^{n}\left(A_{j}, F_{0}^{\prime} \cap A_{j}, F_{2}^{\prime} \cap A_{j}\right)$ is a sum of $R_{2}$-modules of rank $\leq 2$, as desired.

Finally we consider any $R_{2}$-module $\mathbf{F}=\left(F, F_{0}, F_{1}\right)$ with pure $F_{0}$ and $F_{1}$. As above we see that $F_{0} \cap F_{1}$ is a summand in $F$, say $F=F^{\prime} \oplus\left(F_{0} \cap F_{1}\right)$. Clearly,

$$
\mathbf{F}=\left(F^{\prime}, F_{0} \cap F^{\prime}, F_{1} \cap F^{\prime}\right) \oplus\left(F_{0} \cap F_{1}, F_{0} \cap F_{1}, F_{0} \cap F_{1}\right) .
$$

Because the second summand is a direct sum of cyclic $R_{2}$-modules, and $\left(F_{0} \cap F^{\prime}\right) \cap$ $\left(F_{1} \cap F^{\prime}\right)=\left(F_{0} \cap F_{1}\right) \cap F^{\prime}=0$, we may assume that our original representation $\mathbf{F}$ satisfies $F_{0} \cap F_{1}=0$.

Write $F=F_{0} \oplus C$ as above. Then $C=C^{\prime} \oplus\left(F_{1} \cap C\right)$ for some $C^{\prime}$, and we have a decomposition

$$
\mathbf{F}=\left(F_{0} \oplus C^{\prime}, F_{0},\left(F_{0} \oplus C^{\prime}\right) \cap F_{1}\right) \oplus\left(F_{1} \cap C, 0, F_{1} \cap C\right)
$$

in which the second summand is a direct sum of cyclic $R_{2}$-modules.

Consequently, it suffices to replace $\mathbf{F}$ by the $R_{2}$-module $\mathbf{F}^{\prime}=\left(F^{\prime}, F_{0}^{\prime}, F_{1}^{\prime}\right)=\left(F_{0} \oplus\right.$ $\left.C^{\prime}, F_{0},\left(F_{0} \oplus C^{\prime}\right) \cap F_{1}\right)$ above. The proof is complete provided $\mathbf{F}^{\prime}$ satisfies condition (因). To this end, we simply note $F_{0}^{\prime} \cap F_{1}^{\prime} \subseteq F_{0} \cap F_{1}=0$ and $C^{\prime} \cap F_{1}^{\prime} \subseteq C^{\prime} \cap F_{1}=0$.

Suppose $\mathbf{F}=\left(F, F_{0}, F_{1}\right)$ is an $R_{2}$-module as in Corollary 4.6, and write $F=F_{0} \oplus C$. The second paragraph of the proof of Corollary 4.6] shows that $\mathbf{F}$ has a rank 1 direct summand if either intersection $F_{0} \cap F_{1}$ or $C \cap F_{1}$ is nonzero. We use this fact to help establish an analogue to Corollary 4.5 for $R_{2}$-modules. The following result will be applied to Butler groups in Section 5, note that the second submodule $F_{1}$ in Corollary 4.7 is transferred to the third place in the $R_{4}$-module.

Corollary 4.7. Suppose $\mathbf{F}=\left(F, F_{0}, F_{1}\right) \in$ Rep $_{2} R$ has finite rank and $F_{0}, F_{1}$ are pure in $F$. If $\mathbf{F}$ has no rank 1 summands, then there exist pure submodules $F_{2}, F_{3} \subseteq$ $F$ such that $\left(F, F_{0}, F_{2}, F_{1}, F_{3}\right)$ is a complemented, indecomposable representation in $R e p_{4} R$.

Proof. We may decompose $F=F_{0} \oplus C$ since $F_{0}$ is pure in $F$. As remarked above, our hypotheses imply $F_{0} \cap F_{1}=C \cap F_{1}=0$. Put $F_{2}=C$. Because decompositions of the $R_{3}$-module $\mathbf{F}^{\prime}=\left(F, F_{0}, F_{1}, F_{2}\right)$ induce ones of $\mathbf{F}$, it follows that $\mathbf{F}^{\prime}$ has no rank 1 summands. By Corollary 4.5 a fourth pure submodule $F_{3} \subseteq F$ with 
$F_{3} \cap F_{i}=0(i=0,1,2)$ can be adjoined to $\mathbf{F}^{\prime}$ to produce an indecomposable $R_{4}$-module $\left(F, F_{0}, F_{2}, F_{1}, F_{3}\right)$ of the desired form.

Corollary 4.4 shows in particular that indecomposable, complemented $R_{3}$-modules $\left(F, F_{i}: i<3\right)$ of infinite rank need not decompose into direct sums of $R_{3}$-modules of finite rank. A rather obvious necessary condition for such a decomposition to exist is that all quotients $F /\left(F_{i} \oplus F_{j}\right)(i \neq j)$ be direct sums of cyclic $R$-modules. The following example shows that this condition does not suffice.

Example 4.8. Suppose $F_{0}$ and $F_{1}$ are free $R$-modules of rank $\omega$ and the PID $R$ contains infinitely many primes $\left\{p_{i}: i<\omega\right\}$. Then there is a pure submodule $F_{2} \subseteq F=F_{0} \oplus F_{1}$ such that $\mathbf{F}=\left(F, F_{0}, F_{1}, F_{2}\right)$ is a complemented representation in $\operatorname{Rep}_{3} R$, which is not a direct sum of finite rank $R_{3}$-modules. Moreover,

$$
F /\left(F_{0} \oplus F_{2}\right) \cong F /\left(F_{1} \oplus F_{2}\right) \cong \bigoplus_{1 \leq i<\omega} R / p_{i} R
$$

Proof. Write $F_{0}=\bigoplus_{i<\omega} R a_{i}, F_{1}=\bigoplus_{i<\omega} R b$, and denote $d_{i}=a_{i}+b_{i} \in F=F_{0} \oplus F_{1}$. Define

$$
F_{2}=R d_{0} \oplus R\left(b_{0}+p_{1} d_{1}\right) \oplus \bigoplus_{1 \leq i<\omega} R\left(d_{i}+p_{i+1} d_{i+1}\right) .
$$

We first show that $F_{2}$ is pure in $F$. Let $\pi_{a_{i}}: F \longrightarrow R a_{i}$ and $\pi_{b_{i}}: F \longrightarrow R b_{i}$ denote the obvious projections. Suppose $q \in R$ is prime and

$$
x=r d_{0}+s\left(b_{0}+p_{1} d_{1}\right)+\sum_{i \geq 1} r_{i}\left(d_{i}+p_{i+1} d_{i+1}\right)
$$

lies in $F_{2} \cap q F$, where $r, s, r_{i} \in R$.

Then $x \pi_{b_{0}}=(r+s) b_{0}, x \pi_{a_{0}}=r a_{0}$ and $x \pi_{a_{1}}=\left(r_{1}+s p_{1}\right) a_{1}$ are divisible by $q$ in $F$. Therefore $r, s, r_{1} \in q R$. Now $x \pi_{a_{i}}=\left(r_{i-1} p_{i}+r_{i}\right) a_{i}(i \geq 2)$ is in $q F$ as well, hence it follows by induction that $r_{i} \in q R$ for all $i \geq 2$. Therefore $x \in q F_{2}$, and the purity of $F_{2}$ in $F$ is established.

Also note that $F / F_{2} \cong\left(R a_{0} \oplus R b_{0} \oplus \underset{1 \leq i<\omega}{\bigoplus} R d_{i}\right) / F_{2} \oplus \underset{1 \leq i<\omega}{\bigoplus} R a_{i}$ and the first summand is torsion free of rank 1 of type $(1,1, \ldots)$.

It is routine to verify that $\mathbf{F}$ is complemented. Let $\pi_{i}: F \longrightarrow F_{i}$ denote the canonical projection for $i=0,1$. The composition $F_{1} \longrightarrow F \longrightarrow F / F_{0} \oplus F_{2}$ of inclusion followed by the natural epimorphism is surjective with kernel $F_{2} \pi_{1}$, hence $F / F_{0} \oplus F_{2} \cong F_{1} / F_{2} \pi_{1}$. Note $F_{2} \pi_{1}$ is generated by the set

$$
\left\{b_{0}, p_{1} b_{1}\right\} \cup\left\{b_{i}+p_{i+1} b_{i+1}: 1 \leq i<\omega\right\} .
$$

Denote $b_{1}^{\prime}=b_{1}$ and $b_{i}^{\prime}=p_{1} \cdots p_{i-1} b_{i}$ for $i \geq 2$. Because the $p_{k}$ are distinct primes of $R$ it is easy to see that $F_{1}$ is generated by $\left\{b_{i}^{\prime}: 1 \leq i<\omega\right\}$ and $F_{2} \pi_{1}$. Moreover $b_{i}^{\prime}$ has order $p_{i}$ modulo $F_{2} \pi_{1}$, hence

$$
F_{1} / F_{2} \pi_{1}=\bigoplus_{1 \leq i<\omega} R\left(b_{i}^{\prime}+F_{2} \pi_{1}\right) \cong \bigoplus_{1 \leq i<\omega} R / p_{i} R .
$$

Similarly, it follows that $F / F_{1} \oplus F_{2} \cong F_{0} / F_{2} \pi_{0} \cong \underset{1 \leq i<\omega}{\bigoplus} R / p_{i} R$.

Note that if $\mathbf{F}=\left(F, F_{0}, F_{1}, F_{2}\right)$ were a direct sum of finite rank $R_{3}$-modules, $F / F_{2}$ would be a direct sum of finitely generated torsion-free $R$-modules, hence 
free. However a look back at the definition of $F_{2}$ shows $b_{0} \notin F_{2}$, while

$$
b_{0} \equiv-p_{1} d_{1} \quad \bmod F_{2} \quad \text { and } \quad d_{i} \equiv-p_{i+1} d_{i+1} \quad \bmod F_{2}
$$

for all $i \geq 1$. Therefore $0 \neq b_{0}+F_{2} \in \bigcap_{1 \leq i<\omega} p_{i}\left(F / F_{2}\right)$, and $F / F_{2}$ is certainly not a free $R$-module. This finishes 4.8 .

Finally we note an additional property of Example 4.8: Choose an idempotent $\sigma \in$ End $\mathbf{F}$, acting on generators as $a_{i} \sigma=b_{i} \sigma=0$ for $i>1$ and $a_{i} \sigma=a_{1}+p_{2} a_{2}$, $b_{1} \sigma=b_{1}+p_{2} a_{2}$.

Then $d_{1} \sigma=d_{1}+p_{2} d_{2}$ and $\sigma \in$ End $\mathbf{F} \backslash\{0,1\}$. Actually

$$
\begin{aligned}
\mathbf{F}= & \left(\left\langle a_{1}+p_{2} a_{2}, b_{1}+p_{2} b_{2}\right\rangle,\left\langle a_{1}+p_{2} a_{2}\right\rangle,\left\langle b_{1}+p_{2} b_{2}\right\rangle\right) \\
& \oplus\left(\left\langle a_{i}, b_{i}: i>1\right\rangle,\left\langle d_{i}: i>1\right\rangle,\left\langle b_{i}: i>1\right\rangle,\left\langle d_{0}, b_{0}+p_{2} d_{2}, d_{2}+p_{3} d_{3}, \ldots\right\rangle\right)
\end{aligned}
$$

in $R e p_{3} R$, where the second summand $\mathbf{C}_{0}$ is isomorphic to $\mathbf{F}$.

We can find a descending chain of $\operatorname{Rep}_{3} R$-submodules $\mathbf{C}_{i}$ with completely decomposable complements (into summands of ranks 1 and 2) in $\mathbf{F}$ such that $\bigcap_{i \in \omega} \mathbf{C}_{i}=$ $\left\langle a_{0}, b_{0}\right\rangle$.

\section{Applications to Butler Groups}

A Butler group of finite rank is a torsion-free, homomorphic image of any finite direct sum of additive subgroups of the rationals. More generally, a countable torsion-free abelian group is called a Butler group if it is the union of an ascending chain of pure, finite-rank Butler subgroups. There are a number of alternate definitions of Butler groups, many of them as deceptively simple as the ones we just gave. This special class of groups, dazzlingly complex, has fundamental links to several branches of mathematics: ring theory, abelian group and module theory, representation theory, even number theory and category theory. It is one of the links to representation theory that concerns us in this brief section. The methodology we summarize in the following paragraph is due to Arnold and Dugas [5].

Fix an additive subgroup $C$ and a subring $R$ of $\mathbb{Q}$. Our work will revolve around a set $\mathfrak{A}=\left\{A_{0}, A_{1}, A_{2}, \ldots\right\}$ of additive subgroups of $\mathbb{Q}$, each containing 1 , that satisfy the following conditions whenever $i \neq j<\omega$ :

(i) $A_{i} \cap A_{j} \cong C$;

(ii) $\operatorname{Hom}\left(A_{i}, A_{j}\right)=0$;

(iii) $\operatorname{End}\left(A_{i}\right)=R$.

Let $E$ be any set of rational primes (possibly empty) such that for each $p \in E$, $p^{-1}$ is not contained in any member of $\mathfrak{A}$. Moreover, let $\tau_{i}=\operatorname{type}\left(A_{i}\right)$ and $\tau=$ type $(C)$.

With the sets $\mathfrak{A}$ and $E$ in place, we let $B\left(T_{n}, E\right)$ denote the category of finiterank Butler groups $G$ with typesets contained in $T_{n}=\left\{\tau_{0}, \ldots, \tau_{n-1}, \tau\right\}$ such that $G$ modulo the sum $G\left(\tau_{0}\right)+\cdots+G\left(\tau_{n-1}\right)$ of type subgroups is a torsion $E$-group.

The extended category $B\left(T_{n}, E, \omega\right)$ will denote those countable groups which are obtainable as unions of ascending chains of pure subgroups in $B\left(T_{n}, E\right)$. The theory recently developed in [5] states, roughly, that the groups $G$ in $B\left(T_{n}, E, \omega\right)$ are determined up to isomorphism by representations

$$
\mathbf{F}=\left(F, F_{i}: i<n\right) \in \operatorname{Rep}_{n} R, \text { where } A_{i} F_{i}=G\left(\tau_{i}\right)
$$


in the divisible hull of $G$, and $F$ is the $E$-purification of $F_{0}+\cdots+F_{n-1}$ in $G$. The existence and uniqueness of the free $R$-modules $F_{i} \subseteq G$ with the stated property are guaranteed by [5, Lemma 1.1], and the situation is such that:

(a) each $F_{i}$ is pure in the free $R$-module $F$;

(b) $F_{i} \cap F_{j}=0$ if $i \neq j$; and

(c) $F /\left(F_{0}+\cdots+F_{n-1}\right)$ is a torsion $E$-group.

We use

$$
L_{n}: B\left(T_{n}, E, \omega\right) \rightarrow \operatorname{Rep}_{n} R
$$

to denote the functor just described, whose image is the full subcategory of $R e p_{n} R$ comprised of countable-rank representations $\mathbf{F}$ which satisfy the conditions (a), (b) and (c). Theorem 3.1 in [5] asserts that $L_{n}$ is a full, additive, rank-preserving equivalence whose inverse takes

$$
\mathbf{F}=\left(F, F_{i}: i<n\right) \in \operatorname{Im} L_{n} \text { to } G=F+\sum_{i<n} A_{i} F_{i} \subseteq \mathbb{Q} \otimes F .
$$

$G$ is a member of $B\left(T_{n}, E, \omega\right)$. Moreover, $G\left(\tau_{i}\right)$ coincides with the subgroup $A_{i} F_{i}$ used to build $G$.

The following consequence of Corollary 4.6 has been noted in a number of recent papers [5] 9, 28, 22, 46. It provides, in effect, a complete classification of finite-rank Butler groups with at most two critical types.

Proposition 5.1. Groups in $B\left(T_{2}, E\right)$ are direct sums of indecomposable groups isomorphic to $A_{0}, A_{1}$ and $A_{0} e+A_{1} f+R \frac{e+a f}{b} \subseteq \mathbb{Q} e \oplus \mathbb{Q} f$, where $a, b \neq 0$ are coprime in $R$ and $b$ is a nonunit whose prime factors lie in $E$.

On the other hand, there are indecomposable groups in $B\left(T_{3}, E\right)$ of arbitrarily large finite rank, even when $E=\emptyset$ (see [5. Example A.5]). A tractable subclass of $B\left(T_{3}, E\right)$ consists of those members $G$ whose images $L_{3}(G)$ are the complemented $R_{3}$-modules studied earlier in this paper; by Theorem 2.6. Remark 2.7 and the categorical equivalence discussed above, these special Butler groups are direct sums of indecomposable groups isomorphic to $A_{0}, A_{1}, A_{2}$ and $A_{0} e+A_{1} f+A_{2}(a e+b f) \subseteq$ $\mathbb{Q} e \oplus \mathbb{Q} f$, where $a \neq 0 \neq b$ are coprime in $R$. We refer the interested reader to [5] Corollary A.4] for details about the group-theoretical properties of this particular class of Butler groups with three or fewer critical types.

We know of no result quite like the following anywhere in the literature on Butler groups. It is complementary to Proposition 5.1, and asserts that most Butler groups with two critical types are full-rank subgroups of indecomposable Butler groups with only two additional critical types.

Proposition 5.2. If $G \in B\left(T_{2}, E\right)$ has no rank one direct summands, then $G$ is contained in an indecomposable group $H \in B\left(T_{4}, \emptyset\right)$ with $H / G$ torsion. Moreover, $H=\sum_{i<4} H\left(\tau_{i}\right)$ can be chosen so that $H\left(\tau_{i}\right)=G\left(\tau_{i}\right)$ for $i=0,1$, and so that $H / G$ is a homomorphic image of a finite direct sum of copies of $A_{2} / R$ and $A_{3} / R$.

Proof. The assumptions (i)-(iii) we made about the set $\mathfrak{A}$ of rank one groups imply that the PID $R$ contains infinitely many rational primes. Assume $G \in B\left(T_{2}, E\right)$ has no rank one summands, and denote $\mathbf{F}=L_{2}(G)=\left(F, F_{0}, F_{1}\right)$. Then $\mathbf{F}$ satisfies all conditions needed to apply Corollary 4.7, we conclude the existence of pure submodules $F_{2}, F_{3} \subseteq F$ such that $F_{i} \cap F_{j}=0$ if $0 \leq i \neq j<4$, and $\mathbf{F}^{\prime}=\left(F, F_{0}, F_{2}, F_{1}, F_{3}\right)$ is a complemented, indecomposable $R_{4}$-module. Note $F=\sum_{i<4} F_{i}$ because $\mathbf{F}^{\prime}$ is complemented. Applying the inverse of $L_{4}$ to the indecomposable representation 
$\left(F, F_{0}, F_{1}, F_{2}, F_{3}\right)$, we obtain an indecomposable member $H=F+\sum_{i<4} A_{i} F_{i} \subseteq$ $\mathbb{Q} \otimes F$ of $B\left(T_{4}, \emptyset\right)$. If we identify $G$ with $F+A_{0} F_{0}+A_{1} F_{1}=L_{2}^{-1}(\mathbf{F})$, then $G$ is embedded in $H$ with $G\left(\tau_{i}\right)=A_{i} F_{i}=H\left(\tau_{i}\right)$ for $i=0,1$. Clearly, $H / G$ is torsion because $\operatorname{rank}(H)=\operatorname{rank}(F)=\operatorname{rank}(G)$. In fact, since $H=G+H\left(\tau_{2}\right) \oplus H\left(\tau_{3}\right)$ and $F_{2} \oplus F_{3}$ is contained in $G \cap\left(H\left(\tau_{2}\right) \oplus H\left(\tau_{3}\right)\right)$, it follows that

$$
\frac{H}{G} \cong \frac{H\left(\tau_{2}\right) \oplus H\left(\tau_{3}\right)}{G \cap\left(H\left(\tau_{2}\right) \oplus H\left(\tau_{3}\right)\right)}
$$

is a homomorphic image of

$$
\frac{A_{2} F_{2} \oplus A_{3} F_{3}}{F_{2} \oplus F_{3}}
$$

hence has the form we desired.

Finally, we give some examples of Butler groups of infinite rank that arise naturally from the complemented representations of infinite rank constructed in the previous section. For the moment, we assume $R=\mathbb{Z}$, and that $\mathfrak{A}$ has been chosen so that the characteristic of 1 in the group $A_{2}$ consists of only zeros and ones.

Example 5.3. There is a group $G \in B\left(T_{3}, \emptyset, \omega\right)$ of infinite rank such that

(i) $\operatorname{End}(G)=\mathbb{Z}$;

(ii) $G$ modulo the completely decomposable subgroup $G\left(\tau_{0}\right) \oplus G\left(\tau_{1}\right)$ is a direct sum of elementary $p$-groups.

To construct $G$, let $\mathbf{F}=\left(F, F_{0}, F_{1}, F_{2}\right)$ be a rigid, complemented $\mathbb{Z}_{3}$-module, the existence of which was established in Corollary 4.4 Applying the inverse of the (full) functor $L_{3}$ to $\mathbf{F}$, we obtain a rigid group $G=\sum_{i<3} G\left(\tau_{i}\right)$ in $B\left(T_{3}, \emptyset, \omega\right)$. Now since $F=F_{0} \oplus F_{1}$, we reason that

$$
\frac{G}{G\left(\tau_{0}\right) \oplus G\left(\tau_{1}\right)} \cong \frac{G\left(\tau_{2}\right)}{G\left(\tau_{2}\right) \cap\left(G\left(\tau_{0}\right) \oplus G\left(\tau_{1}\right)\right)}=\frac{A_{2} F_{2}}{A_{2} F_{2} \cap\left(A_{0} F_{0} \oplus A_{1} F_{1}\right)}
$$

is a homomorphic image of $A_{2} F_{2} / F_{2} \cong \bigoplus_{\omega} A_{2} / \mathbb{Z}$. Because of our assumption about the characteristic of 1 in $A_{2}$, it follows that the last factor is a direct sum of elementary $p$-groups, establishing (ii).

We remark that rigid extensions $H$ of completely decomposable groups $D=$ $D\left(\tau_{0}\right) \oplus D\left(\tau_{1}\right)$ by torsion groups were previously constructed in [22], but with the important difference that $H / D$ was divisible. The discussion and examples in [28, Section 4] make it clear that different techniques are required to obtain rigid groups $H$ for which $H / D$ is, say, a direct sum of cyclic groups. Our work in Section 4 that led to indecomposable, complemented $\mathbb{Z}_{3}$-modules of infinite rank enabled us to realize the group in Example 5.3 above with relative ease; compare the construction of a similar kind of rigid group given in [28, Examples 2.2 and 5.2]. Depending on the choice of $\mathbf{F}$, however, it can be the case that $G$ modulo the completely decomposable subgroup $G\left(\tau_{1}\right) \oplus G\left(\tau_{2}\right)$ is divisible.

We want to point out the existence of what are still fairly intractable members $G$ of $B\left(T_{3}, \emptyset, \omega\right)$ for which all quotients $G /\left(G\left(\tau_{i}\right) \oplus G\left(\tau_{j}\right)\right)(i \neq j)$ are direct sums of elementary $p$-groups. To do this, we continue to take $R=\mathbb{Z}$, and make a slightly more careful choice of $\mathfrak{A}$. Partition the rational primes into two disjoint, infinite sets $\Pi_{1}$ and $\Pi_{2}$, and choose $\mathfrak{A}$ such that for $i=0,1,2$, the characteristic of 1 in $A_{i}$ consists of zeros and ones, with the latter entries occurring only in places corresponding to primes in $\Pi_{1}$. 
Example 5.4. There is a Butler group $G=\sum_{i<3} G\left(\tau_{i}\right) \in B\left(T_{3}, \emptyset, \omega\right)$ of infinite rank such that:

(i) If $0 \leq i \neq j<3$, then $G$ modulo the completely decomposable subgroup $G\left(\tau_{i}\right) \oplus G\left(\tau_{j}\right)$ is a direct sum of elementary p-groups;

(ii) $G$ is not a direct sum of finite-rank subgroups.

To construct $G$, we invoke Example 4.8 and obtain a complemented $\mathbb{Z}_{3}$-module $\mathbf{F}=\left(F, F_{0}, F_{1}, F_{2}\right)$ of rank $\omega$ such that

$$
F / F_{0} \oplus F_{2} \cong F / F_{1} \oplus F_{2} \cong \bigoplus_{p \in \Pi_{2}} \mathbb{Z} / p \mathbb{Z}
$$

but $\mathbf{F}$ is not a direct sum of finite-rank $\mathbb{Z}_{3}$-modules. Let

$$
G=\sum_{i<3} A_{i} F_{i} \subseteq \mathbb{Q} \otimes F .
$$

Since $L_{3}(G)=\mathbf{F}, G$ is not a direct sum of finite-rank subgroups. Because $\mathbf{F}$ is complemented, it follows as in the proof of Example 5.3 that $G / G\left(\tau_{0}\right) \oplus G\left(\tau_{1}\right)$ is a homomorphic image of $\bigoplus_{\omega} A_{2} / \mathbb{Z}$, hence is a direct sum of elementary $p$-groups. Note that our choice of the sets $\Pi_{1}$ and $\Pi_{2}$ (disjoint) and of the characteristic of 1 in $A_{0}$ implies that $A_{0} F / F_{1} \oplus F_{2}$ is a direct sum of elementary $p$-groups, where $p$ ranges over $\Pi_{2}$ and the places where the characteristic of 1 in $A_{0}$ is nonzero. Since $G / G\left(\tau_{1}\right) \oplus G\left(\tau_{2}\right)$ is naturally a homomorphic image of $A_{0} F / F_{1} \oplus F_{2}$, it is of the desired form. Similarly, the other factor $G / G\left(\tau_{0}\right) \oplus G\left(\tau_{2}\right)$ is an image of $A_{1} F / F_{0} \oplus F_{2}$ and is therefore a direct sum of elementary p-groups, as well.

\section{REFERENCES}

[1] U. Albrecht and P. Hill, Butler groups of infinite rank and axiom 3, Czech. Math. J. 37112 (1987), 293-309. MR 88f:20070

[2] D. Arnold, A class of pure subgroups of completely decomposable abelian groups, Proc. Amer. Math. Soc. 41 (1973), 37-44. MR 48:4152

[3] D. Arnold, Finite rank torsion-free abelian groups and rings, Vol. 931, Lecture Notes in Math., Springer, Berlin 1982. MR 84d:20002

[4] D. Arnold, Representations of partially ordered sets and abelian groups, pp. 91-109 in Abelian Group Theory, Contemporary Mathematics 87, Amer. Math. Soc., Providence, RI 1989. MR 90j:20118

[5] D. Arnold and M. Dugas, Butler groups with finite typesets and free groups with distinguished subgroups, Comm. Algebra 21 (1993), 1947-1982. MR 94c:20090

[6] D. Arnold and M. Dugas, Representations of finite posets and near-isomorphism of finite rank Butler groups, Rocky Mountain J. Math. 25 (1995), 591-609. MR 96g:20071

[7] D. Arnold and C. Vinsonhaler, Endomorphism rings of Butler groups, Journ. Austral. Math. Soc. 42 (1987), 322-329. MR 88f:20069

[8] D. Arnold and C. Vinsonhaler, Finite rank Butler groups: A survey of recent results, pp. 17-39 in Abelian Groups, Lecture Notes in Pure and Applied Mathematics, vol. 146, Marcel Dekker 1993. MR 94c:20089]

[9] K. Benabdallah and M. A. Ouldbeddi, Finite essential extensions of torsion-free abelian groups, Canad. Journ. Math. 48 (1996), 918-929. [MR 97h:20063

[10] L. Bican and L. Salce, Butler groups of infinite rank, pp. 171-189 in Abelian Group Theory, Lecture Notes in Math. 1006, Springer 1983. MR 86c:20050

[11] S. Brenner, Decomposition properties of small diagrams of modules, Sympos. Math. 13 (1974), 127-141. MR 50:13159

[12] S. Brenner, On four subspaces of a vector space, J. Algebra 29 (1974), 587-599. MR 49:7271

[13] S. Brenner and M. C. R. Butler, Endomorphism rings of vector spaces and torsion-free abelian groups, J. London Math. Soc. 40 (1965), 183-187. MR 30:4794]

[14] M. C. R. Butler, A class of torsion-free abelian groups of finite rank, Proc. London Math. Soc. 15 (1965), 680-698. MR 36:1532 
[15] M. C. R. Butler, Torsion-free modules and diagrams of vector spaces, Proc. London Math. Soc. 18 (1968), 635-652. MR 37:6327

[16] M. C. R. Butler, Some almost split sequences in torsion-free abelian group theory, pp. 291-302 in Abelian Group Theory, Gordon and Breach, London 1987. MR 90f:20079

[17] J. Cohen and H. Gluck, Stacked bases for modules over principal ideal domains, J. Algebra 14 (1970), 493-505. MR 40:7241

[18] A. L. S. Corner, Every countable reduced torsion-free ring is an endomorphism ring, Proc. London Math. Soc. (3) 13 (1963), 687-710. MR 27:3704

[19] A. L. S. Corner and R. Göbel, Prescribing endomorphism algebras, a unified treatment, Proc. London Math. Soc. 50 (1985), 447-479. MR 86h:16031

[20] M. Dugas and R. Göbel, Every cotorsion-free algebra is an endomorphism algebra, Math. Zeitschr. 181 (1982), 451-470. MR 84h:13008

[21] M. Dugas and R. Göbel, Endomorphism rings of $B_{2}$-groups of infinite rank, to appear in Israel J. Math. 101 (1997), 141-16. MR 98k:20090

[22] M. Dugas and R. Göbel, Classification of modules with two distinguished pure submodules and bounded quotients, Results in Math. 30 (1996) 264-275. MR 98a:13018

[23] M. Dugas, R. Göbel and W. May, Free modules with two distinguished submodules, Comm. Algebra 25 (1997), 3473-3481. MR 98h:13109

[24] M. Dugas, P. Hill and K.M. Rangaswamy, Infinite rank Butler groups II, Trans. Amer. Math. Soc. 320 (1990), 643-664. MR 90k:20092

[25] M. Dugas and K.M. Rangaswamy, Infinite rank Butler groups, Trans. Amer. Math. Soc. 305 (1988), 129-142. MR 88i:20073

[26] M. Dugas and B. Thomé, Countable Butler groups and vector spaces with four distinguished subspaces, J. Algebra 138 (1991) 249-272. MR 92d:20081

[27] P. Eklof and E. Mekler, Almost free modules, set-theoretic methods, North-Holland, Amsterdam - New York 1990. MR 92e:20001

[28] S. Files and R. Göbel, Gauß' theorem for two submodules, Math. Zeitschr. 228 (1998), 511536. MR 99d:13012

[29] L. Fuchs, Infinite abelian groups, Vol. I, II, Academic Press, New York 1970, 1973. MR 41:333 IMR 50:2362

[30] L. Fuchs and M. Magidor, Butler groups of arbitrary cardinality, Israel Journ. Math. 84 (1993), 239-263. MR 95a:20058

[31] L. Fuchs and C. Metelli, Indecomposable Butler groups of large cardinalities, Archiv Math. 57 (1991), 339-344. MR 92g:20087

[32] I. M. Gelfand and V. A. Ponomarev, Problems of linear algebra and classification of quadruples of subspaces in a finite-dimensional vector space, pp. $163-237$ in: Hilbert Space operators, Tihany 1970, Colloq. Math. János Bolyai, Vol. 5 North-Holland, Amsterdam New York 1972. MR 50:9896

[33] R. Göbel and W. May, Four submodules suffice for realizing algebras over commutative rings, Journ. Pure and Appl. Algebra 65 (1990), 29-43. MR 91j:16036

[34] R. Göbel and W. May, Endomorphism algebras of peak I-spaces over posets of finite prinjective type, Trans. Amer. Math. Soc.. 349 (1997), 3535-3567. MR 97k:16043

[35] E. L. Green and I. Reiner, Integral representations and diagrams, Michigan Math. J. 25 (1978), 53-84; (1988), 312-336. MR 80g:16032

[36] A. W. Hales, Analogues of the stacked bases theorem, pp. 399-405, in Abelian group theory, Vol. 616 Lecture Notes in Math., Springer, Berlin 1977. MR 58:22334

[37] P. Hill, Simultaneous decompositions of an abelian group and a subgroup, Quart. J. Math. Oxford (2) 36 (1985), 425-433. MR 87b:20074

[38] P. Hill and C. Megibben, Generalizations of the stacked bases theorem, Trans. Amer. Math. Soc. 312 (1989), 377-402. MR 89g:20089

[39] I. Kaplansky, Infinite abelian groups, The University of Michigan Press, Ann Arbor, 1969. MR 38:2208

[40] K. J. Krapf and O. Mutzbauer, Classification of almost completely decomposable groups, pp. 151-161 in Abelian groups and modules, Proceed. Udine Conference 1984, Vol. 287, CISM Courses and Lecture Notes, Springer, Wien, 1984. MR 86k:20078]

[41] E. L. Lady, Almost completely decomposable torsion-free abelian groups, Proc. Amer. Math. Soc. 45 (1974), 41-47. MR 50:2366 
[42] E. L. Lady, Nearly isomorphic torsion-free abelian groups, J. Algebra 35 (1975), 235-238. MR 51:5801

[43] W. Lewis, Almost completely decomposable groups with two critical types, Comm. in Algebra 21 (2) (1993), 607-614. MR 94f:20104

[44] A. Mader, Almost completely decomposable abelian groups, book in print at Gordon and Breach, London 1998.

[45] A. Mader, O. Mutzbauer and K. M. Rangaswamy, A generalization of Butler groups, pp. 257275, in Abelian group theory and related topics, Contemporary Math. 171, Amer. Math. Soc. Providence, RI 1994. MR 95h:20070

[46] A. Mader and C. Vinsonhaler, Classifying almost completely decomposable abelian groups, J. Algebra 170 (1994), 754-780. MR 95j:20054

[47] J. Reid, A note on torsion free abelian groups of infinite rank, Proc. Amer. Math. Soc. 13 (1962), 222-225. MR 24:A3190

[48] D. Simson, Linear Representations of Partially Ordered Sets and Vector Space Categories, Algebra, Logic and Applications, Vol. 4, Gordon \& Breach Science Publishers, London 1992. MR 95g:16013

[49] C. Vinsonhaler, A survey of balanced Butler groups and representations, pp. $113-122$ in Abelian groups and modules, Proceed. Colorado Springs 1995, Marcel Dekker, Inc., New York 1996 MR 97k:20091

Department of Mathematics, Wesleyan University, Middletown, Connecticut 06459

E-mail address: sfiles@wesleyan.edu

Fachbereich 6, Mathematik und Informatik, Universität Essen, 45117 Essen, Germany

E-mail address: R.Goebel@Uni-Essen.DE 